\title{
Mối quan hệ giữa quản lý tri thức, đổi mới, và hiệu quả tổ chức: Nghiên cứu thực nghiệm tại các doanh nghiệp vừa và nhỏ ở Việt Nam
}

\section{Relationship between knowledge management, innovation, and organization performance: An empirical study in Vietnamese small and medium-sized enterprises}

\author{
Phạm Anh Tuấn ${ }^{1 *}$, Phạm Quốc Trung ${ }^{2}$ \\ ${ }^{1}$ Trường Đại học Công nghiệp Thực phẩm Thành phố Hồ Chí Minh, Việt Nam \\ ${ }^{2}$ Trường Đại học Bách khoa Thành phố Hồ Chí Minh, Việt Nam \\ *Tác giả liên hệ, Email: tuanpanh@hufi.edu.vn
}

THÔNG TIN

DOI: $10.46223 /$ HCMCOUJS. econ.vi.16.2.614.2021

Ngày nhận: 11/08/2020

Ngày nhận lại: 20/09/2020

Duyệt đăng: 20/11/2020

Tù khóa:

doanh nghiệp vừa và nhỏ; đổi mới; hiệu quả tổ chức; quản lý tri thức

Keywords:

small and medium-sized enterprise; innovation; organization performance; knowledge management

\section{TÓM TẮT}

Nghiên cứu này dựa trên mô hình nghiên cứu của ValdezJuárez, García-Pérez de Lema, và Maldonado-Guzmán (2016) và các lý thuyết có liên quan nhằm mục đính xem xét mối quan hệ giữa Quản Lý Tri Thức (QLTT), đổi mới và Hiệu Quả Tổ Chức (HQTC) trong các Doanh Nghiệp Vừa Và Nhỏ (DNVVN) tại Việt Nam. Bảng câu hỏi khảo sát được gởi tới các nhà quản lý đã và đang làm việc tại các DNVVN thuộc các lĩnh vực xây dựng, thương mại, dịch vụ, và sản xuất. Dũ liệu thu về được phân tích bằng phần mềm SPSS v20 và AMOS v22. Kết quả cho thấy các yếu tố Đào tạo nhân viên, Chiến lược và chính sách của doanh nghiệp, Thu nhận tri thức của nhân viên, và Văn Hóa Tổ Chức (VHTC) là có ảnh hưởng tích cực đến sự đổi mới và sự đổi mới có ảnh hưởng tích cực đến HQTC. Từ kết quả của nghiên cứu này, nghiên cứu cũng đề xuất một số kiến nghị nhằm giúp các DNVVN áp dụng QLTT một cách hiệu quả, từ đó, nâng cao năng lực đổi mới và cải thiện HQTC.

\section{ABSTRACT}

This study is based on the research model of Valdez-Juárez, García-Pérez de Lema, and Maldonado-Guzmán (2016), and related theories aiming to examine the relationship between knowledge management, innovation, and organizational performance in Vietnamese SMEs. The results show that employee training, corporate strategy and policy, acquiring employee's knowledge, and organizational culture have a positive impact on innovation, and innovation positively impacts organizational performance. From the results of this study, the study also proposes a number of recommendations to help SMEs effectively apply knowledge management, thereby improving their capacity to innovate and improve organizational performance. 


\section{Giới thiệu}

Tri thức được coi là một cách tiếp cận chiến lược trong việc tạo ra lợi thế cạnh tranh (Asgarian, 2012). Mặt khác, tri thức có thể là một yếu tố thuận lợi trong việc tạo ra giá trị (Chen, Lin, \& Chang, 2009). Tuy nhiên, tri thức đơn thuần không thể cung cấp những lợi thế nêu trên và cần phải được quản lý. QLTT được đề cập đến như là một nhu cầu cơ bản trong thời đại ngày nay. Trong đó, QLTT là công cụ giúp cho các doanh nghiệp hoạt động hiệu quả hơn, đặc biệt là hỗ trợ cho sự đồi mới (Paez-Logreira, Zamora-Musa, \& Velez-Zapata, 2016). Sự đổi mới đóng vai trò quan trọng trong việc cung cấp các cơ hội để phát triển và vượt qua các đối thủ của nó. QLTT chính là vũ khí quan trọng để duy trì lợi thế cạnh tranh và nâng cao HQTC (Pannu, 2017). QLTT hiệu quả ảnh hưởng tích cực đến khả năng cạnh tranh của tổ chức và sự đổi mới dẫn đến việc cải thiện HQTC (Torabi \& El-Den, 2017).

Như chúng ta đã biết, DNVVN chiếm tỷ lệ rất lớn trong tổng số các doanh nghiệp và đóng vai trò quan trọng trong sự nghiệp phát triển kinh tế - xã hội của hầu hết các quốc gia trên thế giới, là động lực tăng trưởng, là xương sống của nền kinh tế, và chính là động lực của sự đổi mới. Tại Việt Nam, theo kết quả điều tra của Cục Thông tin Khoa học và Công nghệ Quốc gia về hoạt động đổi mới sáng tạo trong các doanh nghiệp. Kết quả cho thấy, hoạt động đổi mới sáng tạo trong các doanh nghiệp nói chung vẫn còn hạn chế, trong khi đó, số lượng DNVVN chiếm hơn $98 \%$ tổng số doanh nghiệp, đóng góp hơn $50 \%$ vào tổng GDP và thuê khoảng $77 \%$ tổng lực lượng lao động. Trong bối cảnh QLTT, các DNVVN đang phải đối mặt với nhiều khó khăn như sự lãng phí tri thức do tỷ lệ thay đổi nhân viên cao, khả năng chia sẻ tri thức trong nhân viên thấp, khó thu hút được những người có tay nghề cao vì không chú trọng đến chế độ đãi ngộ nhân tài, không có cơ chế đào tạo nhân viên, không chú trọng đến việc nghiên cứu và phát triển do hạn chế về mặt tài chính, thiếu nhân lực, công nghệ lạc hậu, quản lý yếu kém, năng lực cạnh tranh yếu, ít đổi mới, và chưa sẵn sàng cho việc hội nhập (Pham, 2016). Bên cạnh đó, khả năng đổi mới của các DNVVN ở Việt Nam khá thấp và tác động thực sự của các quá trình QLTT đến hiệu quả đổi mới của các DNVVN Việt Nam không được đo lường và xác nhận rõ ràng (Pham \& Le, 2018). Điều này đặt ra một thách thức đặc biệt khó khăn đối với các DNVVN vì họ thường thiếu các nguồn lực cần thiết để sử dụng toàn bộ kho tri thức của mình.

Mặc dù QLTT đã được nghiên cứu nhiều nhưng chủ yếu tập trung vào các doanh nghiệp lớn (Roxas, Battisti, \& Deakins, 2014). Bên cạnh đó, ở hầu hết các DNVVN không có QLTT có hệ thống (Wong \& Aspinwall, 2005) và nếu có thì việc thực hiện có thể được coi là kém hiệu quả (Durst \& Edvardsson, 2012). Điều này chứng tỏ, lợi ích của QLTT chưa được các DNVVN khai thác triệt để, chủ đề về QLTT trong các DNVVN là một trong các chủ đề quan trọng nhưng chưa nhận được sự quan tâm đúng mức và việc triển khai thành công QLTT trong các DNVVN rất ít được đề cập đến trong các nghiên cứu trước (Cantú, Criado, \& Criado, 2009; Evangelista, Esposito, Lauro, \& Raffa, 2010; Serenko, 2013). Hơn thế nữa, các chủ đề về nhận dạng tri thức, lưu trữ tri thức và sử dụng tri thức là rất hiếm, các nghiên cứu thực nghiệm hiện tại chỉ cung cấp những hiểu biết rời rạc về QLTT trong các DNVVN (Durst \& Edvardsson, 2012). Mặt khác, QLTT là yếu tố dự báo quan trọng về sự đổi mới và HQTC nhưng chưa được nghiên cứu đầy đủ, đặc biệt hơn là thiếu các nghiên cứu về QLTT ở các nước đang phát triển và cần phải hiểu tác động của thực tiễn và khả năng QLTT trong bối cảnh của các nước đang phát triển (Rashdi, Akmal, \& Al-shami, 2019) như Việt Nam.

Mục tiêu của nghiên cứu này là phân tích thực nghiệm các mối quan hệ giữa QLTT, sự đổi mới và HQTC trong các DNVVN tại Việt Nam. Trong môi trường cạnh tranh toàn cầu như hiện nay, điều quan trọng là các DNVVN áp dụng QLTT một cách hiệu quả, từ đó, nâng cao năng lực đổi mới và cải thiện $\mathrm{HQTC}$. Do đó, nghiên cứu này nhằm mục đích trả lời hai câu hỏi 
sau: Có hay không tác động trực tiếp của QLTT đến sự đổi mới trong các DNVVN? và (2) Liệu sự đổi mới có ảnh hưởng đến HQTC trong các DNVVN không?

Cấu trúc của bài viết được tổ chức như sau: (1) Giới thiệu, (2) Cơ sở lý thuyết và mô hình nghiên cứu; (3) Phương pháp nghiên cứu; (4) Kết quả nghiên cứu; (5) Thảo luận kết quả nghiên cứu; và cuối cùng, (6) Kết luận.

\section{Cơ sở lý thuyết và mô hình nghiên cứu}

\subsection{Co' sở lý thuyết}

\subsubsection{Tri thíc (Knowledge)}

Là "niềm tin được minh chứng là đúng" (Nonaka \& Takeuchi, 1995). Sự tiến hóa của nhận thức luận khoa học đã hình thành cấu trúc thứ bậc từ dữ liệu $=>$ thông tin $=>$ tri thức, theo 2 chiều hướng: sự hiểu biết, và sự độc lập với ngữ cảnh (Serban \& Luan, 2002). Polanyi (1966) đã phân loại tri thức thành hai loại: (1) tri thức ẩn (tacit) trong đầu con người, khó nắm bắt, và (2) tri thức hiện (explicit) có thể biểu diễn, nắm bắt dễ dàng. Trong bối cảnh DNVVN dường như kém tiến bộ về xây dựng tri thức, có cách tiếp cận máy móc hơn đối với khái niệm này, ít dựa vào tương tác xã hội, việc lập kế hoạch và ra quyết định chỉ tập trung vào một người quản lý chủ sở hữu (Durst \& Edvardsson, 2012).

\subsubsection{Quản lý tri thúc (Knowledge management)}

QLTT được định nghĩa theo nhiều cách khác nhau do tính đa chiều của nó (Darroch, 2003). Xác định QLTT là việc xác định và phân tích các tri thức có sẵn và cần thiết để đạt được các mục tiêu của tổ chức (Chawla \& Joshi, 2010). Theo Cohen và Levinthal (2000), QLTT làm tăng năng lực hấp thụ của nhân viên để trở nên sáng tạo hơn. QLTT là cách tiếp cận hệ thống để thu thập, chia sẻ, và sử dụng tri thức trong quy trình để cải thiện HQTC (Augier \& Teece, 2009). Thực tiễn QLTT được đo bởi bốn chiều hướng, gồm: (1) đào tạo; (2) chiến lược và chính sách; (3) thu nhận tri thức; và (4) VHTC (Bozbura, 2007). Từ các lý thuyết trên, nghiên cứu này sẽ xem xét đào tạo, thu nhận tri thức, chiến lược và chính sách, và VHTC là các yếu tố cấu thành của QLTT.

\subsection{3. Đổi mói (Innovation)}

Đổi mới được định nghĩa là “tạo ra tri thức và ý tưởng mới để tạo thuận lợi cho các kết quả kinh doanh mới, nhằm cải thiện các quy trình và cấu trúc kinh doanh nội bộ và tạo ra các sản phẩm và dịch vụ theo định hướng thị trường" (Plessis, 2007). Thuật ngữ đổi mới thường bao gồm ba loại đổi mới: đổi mới sản phẩm, đổi mới quy trình và đổi mới tổ chức (Halila \& Rundquist, 2011). Các nhà quản lý các doanh nghiệp nhỏ có trình độ học vấn cao tập trung chủ yếu vào đồi mới sản phẩm, trong khi các nhà quản lý của các DNVVN tập trung vào sản phẩm, dịch vụ và các quy trình (Chen \& Huang, 2012). Trong nghiên cứu này, sẽ xem xét hai loại đổi mới là đổi mới sản phẩm, dịch vụ và đổi mới quy trình.

Đổi mới sản phẩm được định nghĩa là những thay đổi hoặc tính mới được giới thiệu trong sản phẩm hoặc dịch vụ cuối cùng, trong khi đổi mới quy trình đề cập đến tính mới được giới thiệu trong phương pháp hoặc quy trình sản xuất sản phẩm hoặc dịch vụ (Kim, Kumar, \& Kumar, 2012).

\subsubsection{Hiệu quả tổ chức (Organizational performance)}

HQTC thường được đo lường bằng đầu ra thực tế hoặc kết quả của tổ chức so với đầu ra dự định của nó. Thước đo hiệu quả của doanh nghiệp có thể bao gồm hiệu quả tài chính và hiệu quả kinh tế. Có một sự khác biệt giữa lợi nhuận và hiệu quả, phụ thuộc vào điều kiện thị trường 
và mục tiêu mà các nhà quản lý theo đuổi. Điều đó có nghĩa là hiệu quả cao hơn có thể không nhất thiết chuyển thành lợi nhuận cao hơn. Trong bối cảnh tổ chức, hiệu quả có thể liên kết với tăng trưởng thông qua cải thiện hiệu quả, năng suất, chất lượng, thị phần, ... (Pannu, 2017).

\subsubsection{Doanh nghiẹp vì̀a và nhỏ (Small and medium-sized enterprises)}

Theo Nghị định 39/2018/NĐ-CP, DNVVN là tên gọi tắt của doanh nghiệp siêu nhỏ, nhỏ và vừa được đánh giá theo các tiêu chí sau: số lao động tham gia bảo hiểm xã hội bình quân năm, và tổng doanh thu của năm hoặc tổng nguồn vốn. Trong đó, doanh nghiệp siêu nhỏ có số lao động không quá 10 người và tổng nguồn vốn không quá 03 tỷ đồng; doanh nghiệp nhỏ có số lao động không quá 50 người và tổng nguồn vốn không quá 50 tỷ đồng (thương mại, dịch vụ) hoặc không quá 100 người và không quá 20 tỷ đồng (các lĩnh vực khác); và doanh nghiệp vừa có số lao động không quá 100 người và tổng nguồn vốn không quá 100 tỷ đồng (thương mại, dịch vụ) hoặc không quá 200 người và không quá 100 tỷ đồng (các lĩnh vực khác).

\subsection{Giả thuyết nghiên cứu}

Qua tham khảo các nghiên cứu trước của Cohen và Levinthal (2000), Augier và Teece (2009), Bozbura (2007), và Valdez-Juárez và cộng sự (2016) về mối quan hệ giữa QLTT, đổi mới, và $\mathrm{HQTC}$, mô hình Valdez-Juárez và cộng sự (2016) được kế thừa và áp dụng vì các lý do sau:

- Mô hình của Valdez-Juárez và cộng sự (2016) bao hàm các yếu tố của các nghiên cứu còn lại;

- Chủ đề nghiên cứu về QLTT, đổi mới, và hiệu quả là phù hợp với vấn đề của nghiên cứu này;

- Phù hợp với loại hình doanh nghiệp là các DNVVN, tuy nghiên cứu trước thực hiện ở Tây Ban Nha, nhưng mô hình và thang đo có thể áp dụng cho DNVVN ở Việt Nam. Kết quả nghiên cứu có thể tìm thấy sự khác biệt trong các DNVVN ở hai quốc gia có mức độ phát triển khác nhau;

- Ngoài ra, nghiên cứu trước tập trung vào đổi mới sản phẩm, đổi mới quy trình để cải thiện hiệu quả kinh doanh (Byukusenge, Munene, \& Orobia, 2016) cũng tương đồng với phạm vi đổi mới của nghiên cứu này.

\subsubsection{Mối quan hệ giũa đào tạo và sự đổi mới}

Việc cập nhật các kỹ năng, tri thức mới cho nhân viên sẽ giúp cho họ có thể áp dụng thành công các thay đổi trong công việc và đáp ứng được nhu cầu và nguyện vọng phát triển của người lao động. Tri thức có thể được tạo ra bằng cách đầu tư vào nghiên cứu, đào tạo, phát triển, sáng tạo, chuyển giao, biến đổi, và ứng dụng tri thức (Nikabadi, Bagheri, \& MohammadiHoseini, 2016). Đào tạo nhân viên nhằm cải thiện kỹ năng của họ, cho phép họ thực hiện các nhiệm vụ phức tạp hơn và hoàn thành nhiệm vụ tốt hơn hoặc nhanh hơn. Sáng tạo tri thức thông qua học tập và đào tạo và tri thức được tạo ra có thể được áp dụng cho các sản phẩm, dịch vụ hoặc quy trình kinh doanh mới (Abubakar, Elrehail, Alatailat, \& Elçi, 2017). Từ đó, giả thuyết H1 được phát biểu như sau:

\section{H1: Đào tạo nhân viên có tác động duơng lên sự đổi mới}

\subsubsection{Mối quan hệ giữa chiến luợc, chính sách và sự đổi mới}

Theo Zheng, Yang, và McLean (2010), chiến lược là một trong các yếu tố thành công quan trọng của QLTT. Khi một doanh nghiệp đưa ra được chiến lược và các chính sách bảo vệ việc áp dụng tri thức (Velu, 2015), doanh nghiệp đó có thể đạt được tiêu chuẩn cao về tri thức và 
sự đổi mới (Anderson, Potočnik, \& Zhou, 2014). Các chiến lược và chính sách của QLTT tạo ra nhiều nhân viên sáng tạo hơn, qua đó làm tăng vốn tri thức (Valdez-Juárez et al., 2016). Với chiến lược QLTT rõ ràng, doanh nghiệp có thể đổi mới tốt hơn, đạt được kết quả tài chính tốt hơn, cải thiện quy trình và phát triển khả năng của nguồn nhân lực (Abdi et al., 2018). Từ đó, giả thuyết $\mathrm{H} 2$ được phát biểu như sau:

\section{H2: Chiến lược và chính sách có tác động dương lên sụ đổi mới}

\subsubsection{Mối quan hệ giữa thu nhận tri thức và sụ đổi mói}

Thu nhận tri thức là một giai đoạn quan trọng trong quy trình QLTT. QLTT là một yếu tố cơ bản thúc đẩy sự đổi mới của các DNVVN (Byukusenge \& Munene, 2017). Khi có sự thu nhận tri thức mới, năng lực của nhân viên tăng lên và họ có thể biến đổi tri thức mới và tạo ra cái mới (Chen \& Huang, 2012). Mardani, Nikoosokhan, Moradi, và Doustar (2018) cho rằng các hoạt động của QLTT tác động trực tiếp đến sự đổi mới và gián tiếp thông qua việc tăng khả năng đổi mới. Từ đó, giả thuyết $\mathrm{H} 3$ được phát biểu như sau:

\section{H3: Thu nhận tri thức có tác động dương lên sụ đổi mới}

\subsubsection{Mối quan hê giũa văn hóa tổ chức và sụ đổi mới}

Theo Al-Saifi (2015), "VHTC định hình quá trình tri thức mới được tạo ra và phân phối trong các tổ chức”. Chiến lược đổi mới, cấu trúc tổ chức, và văn hóa đổi mới có tác động tích cực đến hiệu quả đổi mới của doanh nghiệp (Kalay \& Lynn, 2015). VHTC giúp định hình, xác định hành vi của các thành viên và các hoạt động trong tổ chức. Do đó, VHTC có tác động tích cực đến sự đổi mới của tổ chức (Abdi et al., 2018). Từ đó, giả thuyết $\mathrm{H} 4$ được phát biểu như sau:

\section{H4: VHTC có tác động dương lên sự đổi mới}

\subsubsection{Mối quan hệ giũ̃a sụ đổi mới và hiệu quả tổ chức}

Trong một tổ chức, sự đổi mới có thể đóng một vai trò quan trọng trong việc cung cấp các cơ hội để phát triển và vượt qua các đối thủ khác (Sáenz, Aramburu, \& Rivera, 2009). Đổi mới được đánh giá là chiến lược khác biệt hóa quan trọng nhất để có được lợi thế cạnh tranh trên thị trường (Kalay \& Lynn, 2015). Trong đó, đổi mới sản phẩm và đổi mới quy trình là hai yếu tố chiến lược ảnh hưởng đáng kể đến HQTC (Pelser, 2014; Suhag, Solangi, Larik, Lakho, \& Tagar, 2017). Các biện pháp đánh giá HQTC đã được áp dụng từ Lee và Choi (2003) bao gồm nhận thức của các thành viên tổ chức về mức độ thành công chung, thị phần, lợi nhuận, tốc độ tăng trưởng và tính sáng tạo của tổ chức so với các đối thủ chính, đến mức độ tri thức là nâng cao HQTC. Do đó, đổi mới có tác động tích cực đến HQTC (Nikabadi et al., 2016). Từ đó, giả thuyết H5 được phát biểu như sau:

\section{H5: Sụ đổi mới có tác động dương lên HQTC}

\subsubsection{Mối quan hệ giữa quy mô, số năm hoạt động của doanh nghiệp và hiệu quả tổ chức}

Quy mô và số năm hoạt động của doanh nghiệp có tác động tích cực đến sự tồn tại của doanh nghiệp (Park, Shin, \& Kim, 2010). Theo nghiên cứu của Sørensen và Stuart (2000), kinh nghiệm và năng lực tổ chức có được là nhờ số năm hoạt động, nó giúp cho các doanh nghiệp phát triển theo cách hiệu quả hơn. Bên cạnh đó, quy mô của doanh nghiệp có ảnh hưởng tích cực đến đổi mới tổ chức và HQTC vì những doanh nghiệp lớn thường phân bổ nhiều nguồn lực hơn để đầu tư vào đổi mới. Rosenbusch, Brinckmann, và Bausch (2011) đã thực hiện một nghiên cứu với tiêu đề “đổi mới có mang lại lợi nhuận không?”. Mục đích của nghiên cứu này là để có được bằng chứng về vấn đề trong trường hợp nào các doanh nghiệp nhỏ có nguồn lực giới hạn có thể được hưởng lợi từ đổi mới. Các phát hiện cho thấy số năm hoạt động là một trong những yếu tố 
ảnh hưởng đến HQTC. Từ đó, giả thuyết H6 và $\mathrm{H} 7$ được phát biểu như sau:

H6: Có sụ khác biệt về HQTC theo quy mô của doanh nghiẹp

H7: Có sụ khác biệt về HQTC theo số năm hoạt động của doanh nghiệp

\subsection{Mô hình nghiên cứu đề xuất}

Các giả thuyết trên có thể được tóm tắt trong mô hình nghiên cứu sau (Hình 1).

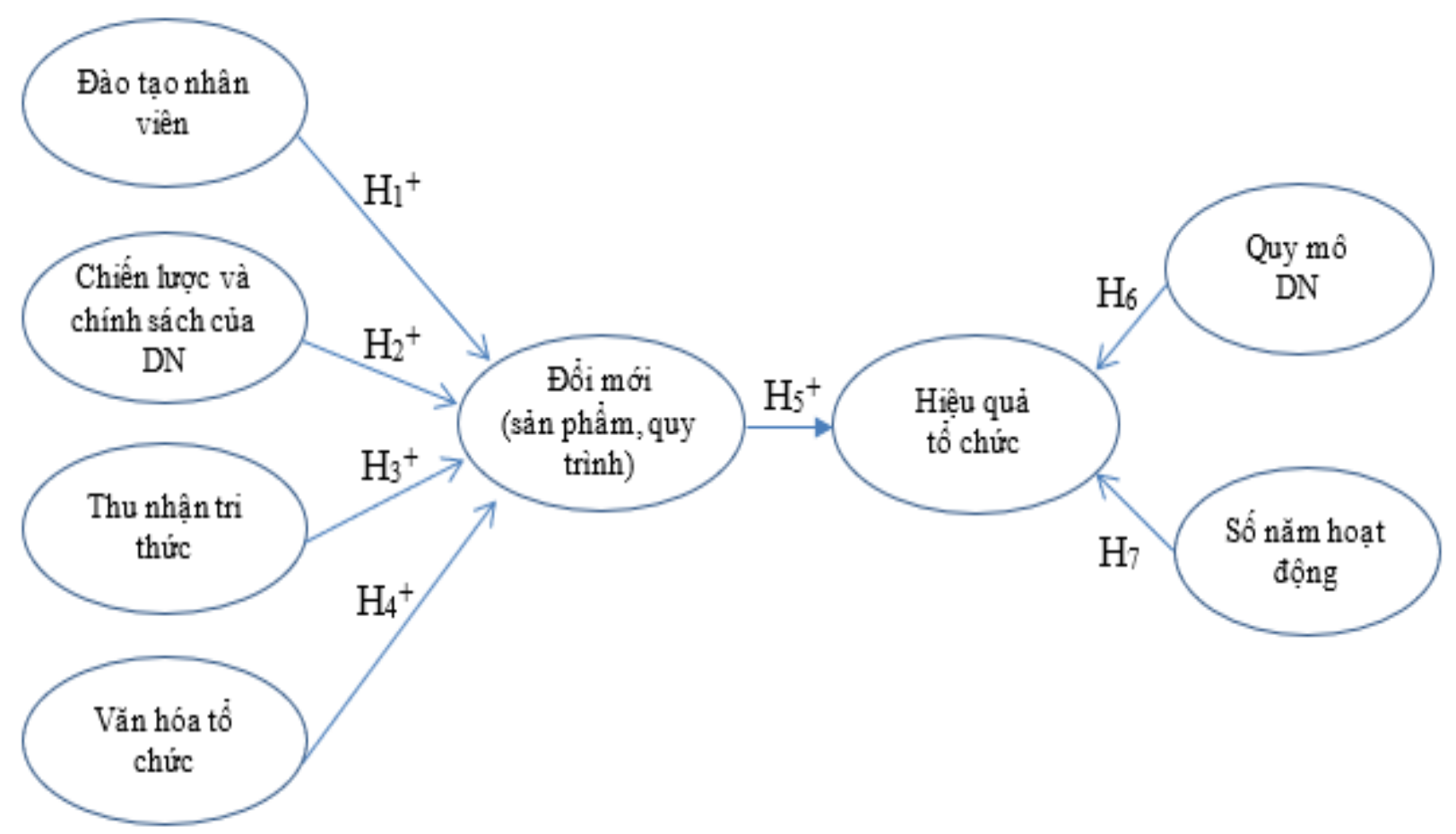

Hình 1. Mô hình nghiên cứu đề xuất

\section{Phương pháp nghiên cứu}

Việc hình thành thang đo được kế thừa từ nghiên cứu của Valdez-Juárez và cộng sự (2016). Trong đó, yếu tố Đào tạo nhân viên (04 biến quan sát), Chiến lược và chính sách (07 biến quan sát), Thu nhận tri thức (05 biến quan sát), VHTC (04 biến quan sát), Đổi mới (04 biến quan sát), và HQTC (03 biến quan sát). Để đảm bảo giá trị nội dung của thang đo, một nghiên cứu định tính thông qua thảo luận tay đôi với một giám đốc, hai trưởng phòng, ba phó phòng và bốn nhân viên quản lý bộ phận/ nhóm tại một số DNVVN ở Việt Nam. Sau đó, thang đo được hiệu chỉnh bằng cách bỏ bớt yếu tố xu hướng (thu nhập từ các hoạt động và số lượng nhân viên trung bình của năm hiện tại so với hai năm trước đó) và chỉnh sửa từ ngữ cho phù hợp với bối cảnh nghiên cứu. Thang đo được sử dụng trong nghiên cứu là thang đo Likert 5 điểm.

Mẫu được chọn theo phương pháp thuận tiện. Bảng câu hỏi khảo sát được gởi tới các nhà quản lý đã và đang làm việc tại các DNVVN thuộc các lĩnh vực xây dựng, thương mại, dịch vụ, và sản xuất tại Việt Nam. Khảo sát này được tiến hành vào tháng $03 / 2020$, kết quả thu về được 227 phiếu khảo sát online và 58 phiếu khảo sát giấy, sau khi đã loại bỏ 74 phiếu không hợp lệ, còn lại 211 phiếu hợp lệ, chiếm tỷ lệ 74.04\%. Dữ liệu được phân tích bằng phần mềm SPSS v20 và $A M O S$ v22 để đánh giá độ tin cậy của thang đo, phân tích nhân tố khám phá (EFA), phân tích nhân tố khẳng định (CFA) và phân tích mô hình cấu trúc tuyến tính (SEM) để kiểm định giả thuyết. Đồng thời, phân tích ANOVA được sử dụng để kiểm định ảnh hưởng của các biến định tính lên các mối quan hệ này. 


\section{Kết quả nghiên cứu}

\subsection{Thống kê mô tả}

Từ kết quả thu thập dữ liệu, có tất cả 211 phiếu trả lời hợp lệ. Thông tin mô tả chi tiết của mẫu khảo sát được trình bày trong Bảng 1 .

\section{Bảng 1}

Mô tả mẫu khảo sát

\begin{tabular}{|c|c|c|c|c|c|}
\hline Thông tin mẫu & Tần suất & Tỷ $1 \hat{\mathrm{e}}(\%)$ & Thông tin mẫu & Tần suất & Tỳ $1 \hat{\mathrm{e}}(\%)$ \\
\hline \multicolumn{3}{|c|}{ Đô tuổi } & \multicolumn{3}{|c|}{ Số năm công tác } \\
\hline Tù̀ $18-35$ & 97 & 46.0 & Dưới 5 năm & 85 & 40.3 \\
\hline Tù $36-45$ & 73 & 34.6 & Tù̀ $5-10$ năm & 63 & 29.9 \\
\hline Trên 45 & 41 & 19.4 & Trên 10 năm & 63 & 29.9 \\
\hline \multicolumn{3}{|c|}{ Trinh đ̇ô văn hóa } & \multicolumn{3}{|c|}{ Số năm hoat å̄ông } \\
\hline Trung cấp & 6 & 2.8 & Từ $1-5$ năm & 31 & 14.7 \\
\hline Cao đẳng & 21 & 10.0 & Từ 6 - 10 năm & 35 & 16.6 \\
\hline Đại học & 132 & 62.6 & Từ $11-15$ năm & 45 & 21.3 \\
\hline Sau đại học & 52 & 24.6 & Trên 15 năm & 100 & 47.4 \\
\hline \multicolumn{3}{|c|}{ Loai hình doanh nghiêp } & \multicolumn{3}{|c|}{ Chisc vu } \\
\hline DN siêu nhỏ & 49 & 23.2 & Ban giám đốc & 42 & 19.9 \\
\hline DN nhỏ & 84 & 39.8 & Trường/ phó phòng & 75 & 35.5 \\
\hline DN vừa & 78 & 37.0 & Nhân viên quản lý & 94 & 44.5 \\
\hline
\end{tabular}

Nguồn: Kết quả phân tích dữ liệu của nhóm nghiên cứu

\begin{tabular}{|c|c|c|}
\hline Thông tin mẫu & Tần suất & Tỷ lệ (\%) \\
\hline \multicolumn{3}{|c|}{ Linh vucc hoat đơng } \\
\hline Thuong mai & 44 & 20.9 \\
\hline Dich vu & 50 & 23.7 \\
\hline San xuat & 29 & 13.7 \\
\hline Xay dung & 15 & 7.1 \\
\hline Thuong mai - Dich vu & 42 & 19.9 \\
\hline Thuong mai - San xuat & 11 & 5.2 \\
\hline Thuong mai - Dich vu - San xuat & 14 & 6.6 \\
\hline Thuong mai - xay dung & 3 & 1.4 \\
\hline Thuong mai - dich vu - xay dung & 3 & 1.4 \\
\hline
\end{tabular}

\section{2. Đánh giá độ tin cậy của thang đo và phân tích EFA}

\subsection{1. Đánh giá độ tin cậy của thang đo}

Việc kiểm định Cronbach's Alpha được đánh giá dựa trên hệ số Alpha nếu loại biến, cùng với hệ số tương quan biến tổng. Nếu một biến đo lường có hệ số tương quan biến tổng $\geq$ 0.3 thì biến đó đạt yêu cầu (Nunnally, 1978).

\section{Bảng 2}

Kết quả phân tích độ tin cậy Cronbach's Alpha

\begin{tabular}{|l|l|c|}
\hline \multicolumn{1}{|c|}{ Thang đo } & \multicolumn{1}{|c|}{ Các biến quan sát } & Hệ số Alpha \\
\hline DAOTAO & DAOT1, DAOT2, DAOT3, DAOT4 & 0.861 \\
\hline CHINHSACH & CHINH1, CHINH2, CHINH3, CHINH4 & 0.824 \\
\hline THUNNHANTRITHUC & THUN1, THUN2, THUN3, THUN4, THUN5 & 0.769 \\
\hline VANHOA & VANH1, VANH2, VANH3 & 0.831 \\
\hline DOIMOI & DOIM1, DOIM2, DOIM3, DOIM4 & 0.834 \\
\hline HIEUQUA & HIEUQ1, HIEUQ2, HIEUQ3 & 0.741 \\
\hline
\end{tabular}

Nguồn: Kết quả xử lý từ dữ liệu điều tra 
Sau khi phân tích Cronbach's Alpha kiểm định độ tin cậy của 06 thang đo với 23 biến quan sát. Kết quả cho thấy, các thang đo đều có hệ số Cronbach's Alpha $>0.6$ và các hệ số tương quan biến tổng đều lớn hơn 0.3 nên các thang đo của các nhóm nhân tố đều đạt độ tin cậy và tiếp tục đưa vào phân tích nhân tố.

\subsubsection{Phân tích nhân tố khám phá EFA cho các biến độc lập và biến phụ thuộc}

Kết quả EFA, sử dụng phương pháp phân tích nhân tố Principal axis factoring và phép quay góc Promax để tìm ra các nhân tố đại điện cho các thang đo. Hệ số KMO được dùng để xem xét sự thích hợp của phân tích nhân tố. Trị số $0.5 \leq \mathrm{KMO} \leq 1$ và sig. $<0.05$ là đủ điều kiện phân tích nhân tố (Hoang \& Chu, 2008).

\section{Bảng 3}

Kết quả phân tích nhân tố đối với các biến độc lập và biến phụ thuộc

\begin{tabular}{|c|c|c|c|c|c|c|c|}
\hline \multirow{2}{*}{ Mã hóa } & \multirow{2}{*}{ Biến quan sát } & \multicolumn{6}{|c|}{ Nhân tố } \\
\hline & & 1 & 2 & 3 & 4 & 5 & 6 \\
\hline DAOT1 & Thường xuyên tổ chức các khóa đào tạo chính thức & 839 & & & & & \\
\hline DAOT2 & $\begin{array}{l}\text { Thường xuyên tồ chức các khóa đào tạo không chính } \\
\text { thức }\end{array}$ & .664 & & & & & \\
\hline DAOT3 & Thường xuyên thực hành tư vấn chính thức & .739 & & & & & \\
\hline DAOT4 & Khuyến khích nhân viên học tập nâng cao trình độ & .880 & & & & & \\
\hline CHINH1 & Thường xuyên thực hiện các ý tưởng mới & & 687 & & & & \\
\hline $\mathrm{CHINH} 2$ & Không ngừng hỗ trợ phát triển các ý tưởng & & .752 & & & & \\
\hline CHINH3 & Cho phép truy cập nhanh thông tin & & .815 & & & & \\
\hline CHINH4 & Thiết lập các thủ tục để hỗ trợ sự đồi mới & & .629 & & & & \\
\hline THUN1 & Thu nhận tri thức từ các nguồn lực công nghiệp khác & & & .731 & & & \\
\hline THUN2 & $\begin{array}{l}\text { Thu nhận tri thức từ các cơ quan công và trung tâm } \\
\text { nghiên cứu }\end{array}$ & & & .757 & & & \\
\hline THUN3 & Thu nhận tri thức từ các đối tác bên ngoài & & & .630 & & & \\
\hline THUN4 & Thu nhận tri thức từ internet & & & .502 & & & \\
\hline VANH1 & Khuyến khích chuyển giao kinh nghiệm & & & & .780 & & \\
\hline VANH2 & $\begin{array}{l}\text { Thiết lập hệ thống giá trị và quảng bá văn hóa trong } \\
\text { toàn đơn vị }\end{array}$ & & & & .819 & & \\
\hline VANH3 & Thúc đẩy làm việc theo nhóm & & & & .790 & & \\
\hline DOIM1 & Thay đồi hoặc cải tiến các sản phẩm/ dịch vụ hiện có & & & & & .597 & \\
\hline DOIM2 & Tiếp thị sản phẩm/ dịch vụ mới & & & & & .818 & \\
\hline DOIM3 & Thay đồi hoặc cải tiến trong quá trình sản xuất / dịch vụ & & & & & .824 & \\
\hline DOIM4 & Cải tiến thiết bị sản xuất đề sản xuất sản phẩm mới & & & & & .760 & \\
\hline HIEUQ1 & Tăng thị phần & & & & & & .850 \\
\hline HIEUQ2 & Tăng lợi nhuận & & & & & & .693 \\
\hline HIEUQ3 & Tăng năng suất & & & & & & .572 \\
\hline
\end{tabular}

Nguồn: Kết quả xử lý từ dữ liệu điều tra

Kết quả phân tích EFA cho thấy, 22 biến quan sát còn lại đều có hệ số tải nhân tố lớn hơn 0.5 và gom thành 06 nhân tố. Kế đến, tiến hành phân tích CFA và SEM.

\subsection{Phân tích nhân tố khẳng định CFA và SEM}

\subsubsection{Phân tích nhân tố khẳng định $C F A$}

Tiêu chuẩn để kiểm định độ tin cậy, tính hội tụ, và tính phân biệt của các thang đo, sử dụng hệ số tải chuẩn hóa (Standardized Loading Estimates) $\geq 0.5$ và độ tin cậy tổng hợp $\mathrm{CR}$ (Composite Reliability) $\geq 0.7$; phương sai trích trung bình AVE (Average Variance Extracted) $\geq$ 
0.5; và phương sai riêng lớn nhất MSV (Maximum Shared Variance) < AVE (Hair và cộng sự, 2010). Một lần nữa hệ số tin cậy tổng hợp và phương sai trích được thực hiện, kết quả là biến THUN4 bị loại bỏ để cải thiện độ phù hợp của mô hình.

Kiểm định CFA sau khi loại biến THUN4, mô hình gồm 06 thang đo khái niệm và 21 biến quan sát. Kết quả CFA được tóm tắt trong Bảng 4 cho thấy, mô hình hiện tại phù hợp với dữ liệu nghiên cứu.

\section{Bảng 4}

Kết quả phân tích CFA

\begin{tabular}{|c|c|}
\hline Các chỉ số đánh giá & Giá trị \\
\hline CMIN/DF & 2.053 \\
\hline GFI & 0.869 \\
\hline TLI & 0.909 \\
\hline CFI & 0.927 \\
\hline RMSEA & 0.071 \\
\hline p & 0.00 \\
\hline
\end{tabular}

Nguồn: Kết quả xử lý từ dữ liệu điều tra

Chi-square=349.021; $d f=170 ; P=.000 ;$

Chi-square/df=2.053;

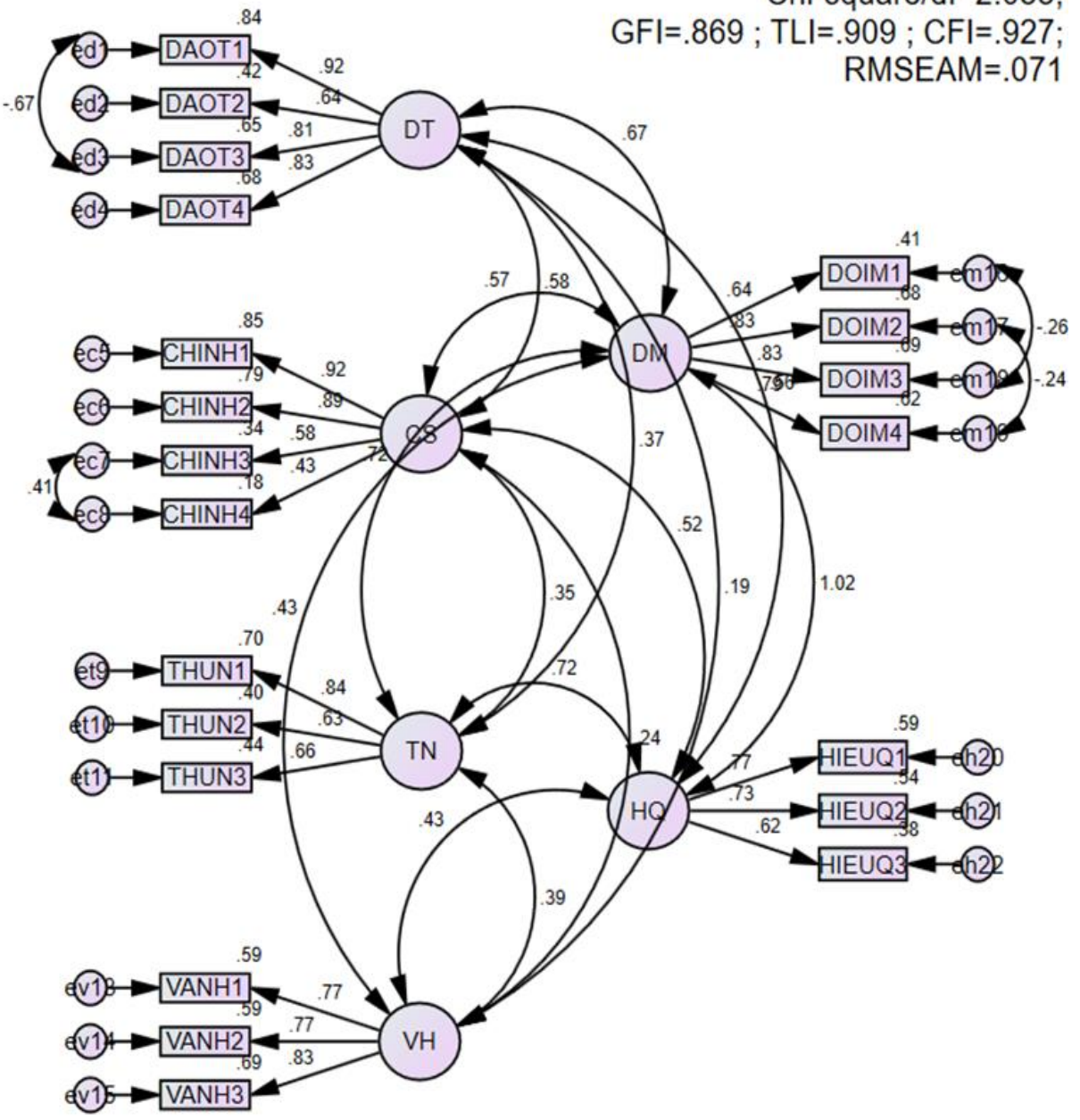

Hình 2. Mô hình thang đo 
Kết quả đo lường sau khi loại bỏ biến quan sát THUN4 được tổng hợp trong Bảng 5:

\section{Bảng 5}

Kết quả đo lường độ tin cậy, tính hội tụ, và tính phân biệt

\begin{tabular}{|c|c|c|c|c|c|c|c|c|}
\hline & CR & AVE & VH & DT & CS & TN & DM & HQ \\
\hline VH & 0.833 & 0.625 & 0.791 & & & & & \\
\hline DT & 0.878 & 0.647 & 0.186 & 0.804 & & & & \\
\hline CS & 0.813 & 0.541 & 0.243 & 0.580 & 0.735 & & & \\
\hline TN & 0.756 & 0.512 & 0.394 & 0.371 & 0.349 & 0.716 & & \\
\hline DM & 0.857 & 0.601 & 0.430 & 0.670 & 0.575 & 0.724 & 0.775 & \\
\hline HQ & 0.753 & 0.505 & 0.431 & 0.563 & 0.523 & 0.718 & 1.022 & 0.711 \\
\hline
\end{tabular}

Nguồn: Kết quả xử lý từ dữ liệu điều tra

Bảng 5 cho thấy giá trị độ tin cậy tổng hợp CR của các biến đều cao hơn 0.7 . Như vậy, độ tin cậy tổng hợp của các thang đo này tốt và được chấp nhận. Phương sai trích bình quân AVE của các thang đo đơn hướng đều lớn hơn 0.5 . Như vậy, phương sai trích của các thang đo này đạt chuẩn và được chấp nhận. Từ các chỉ số trên, mô hình thang đo đảm bảo độ tin cậy. Hệ số mỗi nhân tố đều lớn hơn 0.7 nên đạt độ giá trị phân biệt. Bước tiếp theo, chuyển từ mô hình thang đo sang mô hình nghiên cứu để tiến hành thực hiện kiểm định mô hình SEM.

\subsubsection{Mô hình cấu trúc tuyến tính SEM}

Kết quả phân tích SEM

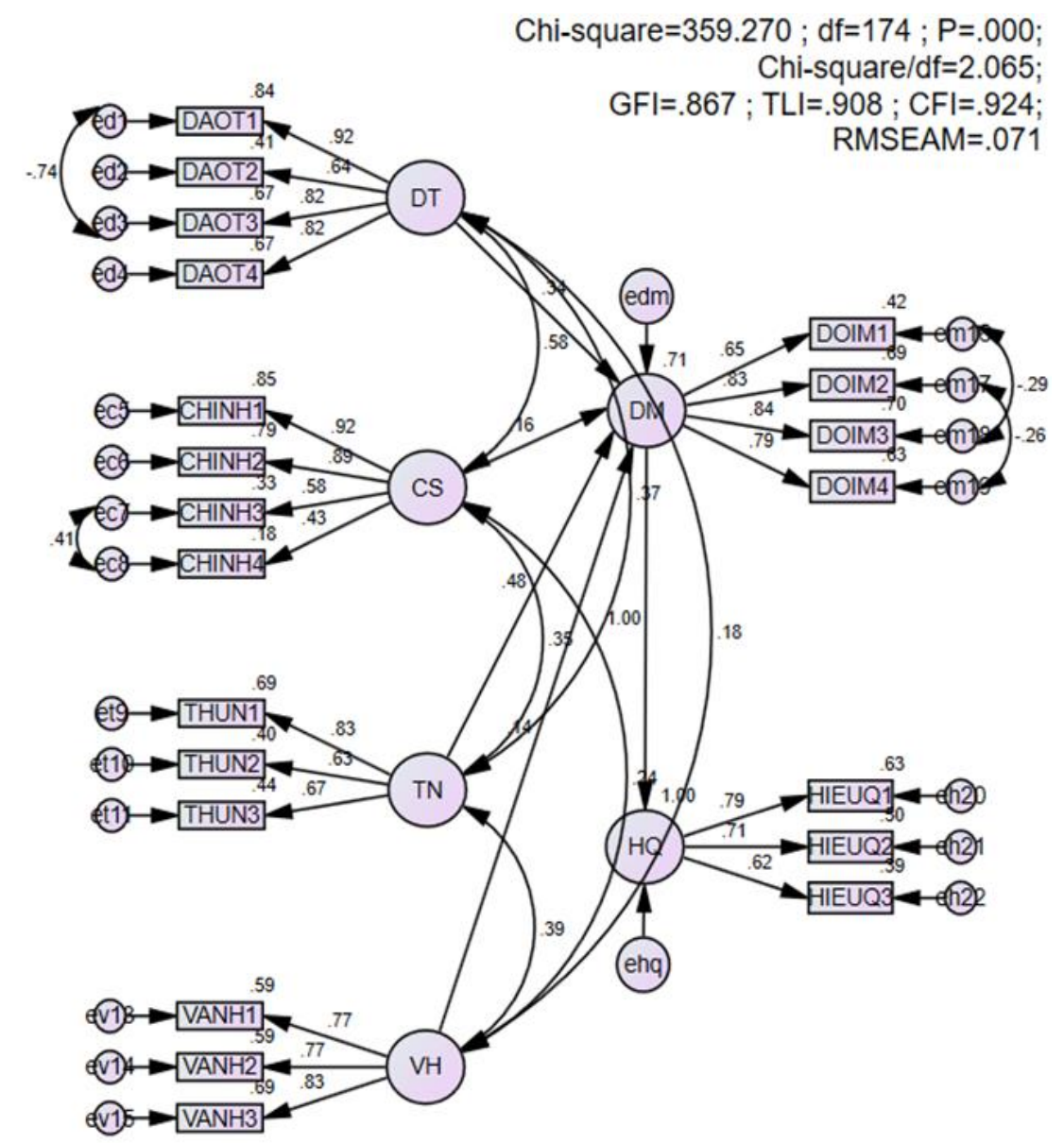

Hình 3. Mô hình SEM chuẩn hóa 
Kết quả phân tích SEM được tóm tắt trong Bảng 6 cho thấy mô hình phân tích phù hợp với dữ liệu nghiên cứu.

\section{Bảng 6}

Kết quả phân tích SEM

\begin{tabular}{|c|c|}
\hline Các chỉ số đánh giá & Giá trị \\
\hline CMIN/DF & 2.053 \\
\hline GFI & 0.869 \\
\hline TLI & 0.909 \\
\hline CFI & 0.927 \\
\hline RMSEA & 0.071 \\
\hline p & 0.00 \\
\hline
\end{tabular}

Nguồn: Kết quả xử lý từ dữ liệu điều tra

Hệ số ước lượng hồi quy chuẩn hóa được tóm tắt trong Bảng 7:

\section{Bảng 7}

Hệ số hồi quy chuẩn hóa

\begin{tabular}{|l|l|l|r|r|r|r|r|}
\hline \multicolumn{2}{|c|}{ Parameter } & Estimate & Lower & Upper & P & Đánh giá \\
\hline DM & $<--$ & TN & .482 & .338 & .624 & .004 & Chấp nhận \\
\hline DM & $<---$ & VH & .139 & .004 & .253 & .047 & Chấp nhận \\
\hline DM & $<--$ & DT & .340 & .209 & .483 & .004 & Chấp nhận \\
\hline DM & $<--$ & CS & .163 & .006 & .302 & .046 & Chấp nhận \\
\hline HQ & $<---$ & DM & 1.001 & .954 & 1.045 & .004 & Chấp nhận \\
\hline
\end{tabular}

Nguồn: Kết quả xử lý từ dữ liệu điều tra

Nhận xét: Mô hình trên có thể nói là phù hợp với dữ liệu nghiên cứu. Với độ tin cậy $95 \%$ :

- Hệ số hồi quy của DT lên DM ước lượng rơi vào khoảng [0.209 - 0.483]

- Hệ số hồi quy của CS lên DM ước lượng rơi vào khoảng [0.006 - 0.302]

- Hệ số hồi quy của TN lên DM ước lượng rơi vào khoảng [0.338 - 0.624]

- Hệ số hồi quy của VH lên DM ước lượng rơi vào khoảng [0.004 - 0.253]

- Hệ số hồi quy của DM lên HQ ước lượng rơi vào khoảng [0.954 - 1.045]

\subsection{Kiểm dịnh ANOVA}

4.4.1. Kiểm định Post-Hoc giũua hai biến LHDN và HIEUQ

Bảng 8.

Kết quả kiểm định Post-Hoc giữa loại hình doanh nghiệp và hiệu quả được tóm tắt trong

\section{Bảng 8}

Post Hoc Tests Multiple Comparisons

\begin{tabular}{|c|c|c|c|c|c|c|}
\hline \multicolumn{7}{|c|}{ Multiple Comparisons } \\
\hline \multicolumn{7}{|c|}{$\begin{array}{l}\text { Dependent Variable: HIEUQ } \\
\text { LSD }\end{array}$} \\
\hline \multirow[t]{2}{*}{ (I) LHDN } & \multirow[t]{2}{*}{ (J) LHDN } & \multirow{2}{*}{$\begin{array}{l}\text { Mean Difference } \\
\text { (I-J) }\end{array}$} & \multirow[t]{2}{*}{ Std. Error } & \multirow[t]{2}{*}{ Sig. } & \multicolumn{2}{|c|}{$95 \%$ Confidence Interval } \\
\hline & & & & & Lower Bound & Upper Bound \\
\hline \multirow{2}{*}{$\begin{array}{l}\text { DN sieu } \\
\text { nho }\end{array}$} & DN nho & $-.33617^{*}$ & 10660 & .002 & -.5463 & -.1260 \\
\hline & DN vua & $-.45735^{\circ}$ & 10810 & .000 & -.6705 & -.2442 \\
\hline \multirow{2}{*}{ DN nho } & DN sieu nho & $.33617^{*}$ & .10660 & .002 & .1260 & .5463 \\
\hline & DN vua & -.12118 & .09325 & .195 & -.3050 & .0627 \\
\hline \multirow{2}{*}{ DN vua } & DN sieu nho & $.45735^{\circ}$ & .10810 & .000 & .2442 & .6705 \\
\hline & DN nho & .12118 & .09325 & .195 & -.0627 & .3050 \\
\hline
\end{tabular}

Nguồn: Kết quả xử lý từ dữ liệu điều tra 
Từ kết quả ở Bảng 8 cho thấy, giữa nhóm doanh nghiệp vừa và siêu nhỏ, doanh nghiệp nhỏ và siêu nhỏ có sự khác biệt có ý nghĩa, tuy nhiên, giữa doanh nghiệp vừa và doanh nghiệp nhỏ không có sự khác biệt có ý nghĩa thống kê (sig. > 0.05). Trong đó, cột Mean Difference (I-J) của nhóm doanh nghiệp vừa và siêu nhỏ có giá trị cao nhất là $0<.45735$, chứng tỏ HQTC của doanh nghiệp vừa lớn hơn đáng kể so với doanh nghiệp siêu nhỏ.

\subsubsection{Kiểm định kiểm định Post-Hoc giũua hai biến SNHD và HIEUQ}

Kết quả kiểm định Post-Hoc giữa số năm hoạt động và hiệu quả được tóm tắt trong Bảng 9.

\section{Bảng 9}

Post Hoc Tests Multiple Comparisons

\begin{tabular}{|c|c|c|c|c|c|c|}
\hline \multicolumn{7}{|c|}{ Multiple Comparisons } \\
\hline \multicolumn{7}{|c|}{$\begin{array}{l}\text { Dependent Variable: HIEUQ } \\
\text { LSD }\end{array}$} \\
\hline \multirow[t]{2}{*}{ (I) $\mathrm{SNHD}$} & \multirow[t]{2}{*}{ (J) SNHD } & \multirow{2}{*}{$\begin{array}{c}\text { Mean Difference } \\
\text { (I-J) }\end{array}$} & \multirow[t]{2}{*}{ Std. Error } & \multirow[t]{2}{*}{ Sig. } & \multicolumn{2}{|c|}{$95 \%$ Confidence Interval } \\
\hline & & & & & \begin{tabular}{l|} 
Lower \\
Bound
\end{tabular} & $\begin{array}{l}\text { Upper } \\
\text { Bound }\end{array}$ \\
\hline \multirow{3}{*}{$\begin{array}{l}\text { Tu } 1-5 \\
\text { nam }\end{array}$} & Tu $6-10$ nam & $.29555^{*}$ & .14677 & .045 & .0062 & .5849 \\
\hline & Tu $11-15$ nam & -.19546 & .13890 & .161 & -.4693 & .0784 \\
\hline & Tren 15 nam & -.15731 & .12233 & .200 & -.3985 & .0839 \\
\hline \multirow{3}{*}{$\begin{array}{l}\text { Tu } 6-10 \\
\text { nam }\end{array}$} & Tu $1-5$ nam & $-.29555^{*}$ & .14677 & .045 & -.5849 & -.0062 \\
\hline & Tu $11-15$ nam & $-.49101^{*}$ & .13412 & .000 & -.7554 & -.2266 \\
\hline & Tren 15 nam & $-.45286^{*}$ & .11687 & .000 & -.6833 & -.2224 \\
\hline \multirow{3}{*}{$\begin{array}{l}\text { Tu } 11-15 \\
\text { nam }\end{array}$} & Tu $1-5$ nam & .19546 & .13890 & .161 & -.0784 & .4693 \\
\hline & Tu $6-10$ nam & $49101^{*}$ & .13412 & .000 & .2266 & .7554 \\
\hline & Tren 15 nam & .03815 & .10682 & .721 & -.1724 & .2487 \\
\hline \multirow{3}{*}{$\begin{array}{l}\text { Tren } 15 \\
\text { nam }\end{array}$} & Tu $1-5$ nam & .15731 & .12233 & .200 & -.0839 & .3985 \\
\hline & Tu $6-10$ nam & $.45286^{*}$ & .11687 & .000 & .2224 & .6833 \\
\hline & Tu $11-15$ nam & -.03815 & .10682 & .721 & -.2487 & .1724 \\
\hline
\end{tabular}

Nguồn: Kết quả xử lý từ dữ liệu điều tra

Từ kết quả ở Bảng 9 cho thấy, giữa các nhóm doanh nghiệp hoạt động từ 01 - 05 năm và từ 06 - 10 năm, từ $06-10$ năm và từ $11-15$ năm, và từ 06 - 10 năm và trên 15 năm là có sự khác biệt có ý nghĩa, tuy nhiên, giữa các nhóm doanh nghiệp hoạt động từ $01-05$ năm và từ $11-15$ năm, từ 01 - 05 năm và trên 15 năm, và từ 11 - 15 năm và trên 15 năm không có sự khác biệt có ý nghĩa thống kê (sig. > 0.05). Trong đó, cột Mean Difference (I-J) của nhóm từ 60-10 năm và từ 11 - 15 năm có giá trị cao nhất, chứng tỏ HQTC của nhóm 11 - 15 năm cao hơn đáng kể so với nhóm 6 - 10 năm. Theo kết quả này, HQTC được sắp theo thứ tự tăng dần như sau: 06 - 10 năm, 01 - 05 năm, trên 15 năm, và 11 - 15 năm.

Tóm lại: Dựa trên các kết quả phân tích trên, các giả thuyết được chấp nhận:

- H1: Có mối quan hệ tích cực giữa đào tạo nhân viên và sự đổi mới

- H2: Có mối quan hệ tích cực giữa chiến lược, chính sách và sự đổi mới

- H3: Có mối quan hệ tích cực giữa thu nhận tri thức và sự đổi mới

- H4: Có mối quan hệ tích cực giữa VHTC và sự đổi mới

- H5: Có mối quan hệ tích cực giữa sự đổi mới và HQTC

- H6: Có sự khác biệt về HQTC theo quy mô của doanh nghiệp

- H7: Có sự khác biệt về HQTC theo số năm hoạt động của doanh nghiệp 


\section{Thảo luận kết quả nghiên cứu}

Các nghiên cứu trước đã chỉ ra rằng, QLTT giúp cải thiện khả năng của các doanh nghiệp trong việc giải quyết vấn đề, thích nghi với những thay đổi và duy trì lợi thế cạnh tranh. Ba thực tiễn chính của QLTT có tác động tích cực đến thành công kinh doanh, bao gồm: văn hóa, chính sách, và thu nhận tri thức (Pham \& Nguyen, 2017). Trong môi trường cạnh tranh cao, đổi mới là chìa khóa thiết yếu cho một doanh nghiệp có được một vị trí có ưu thế hơn và thu được lợi nhuận cao hơn (Kalay \& Lynn, 2015). Bên cạnh đó, Sethibe và Steyn (2016) cũng chỉ ra rằng, HQTC bị ảnh hưởng bởi sự đổi mới, vì vậy, tạo điều kiện cho sự đổi mới là một chức năng quản lý quan trọng có ảnh hưởng trực tiếp đến HQTC. Các kết quả của nghiên cứu này nhằm củng cố thêm mối quan hệ giữa QLTT, sự đổi mới, và HQTC.

Kết quả của nghiên cứu này cho thấy, đổi mới trong các DNVVN ở Việt Nam chịu tác động của các yếu tố theo thứ tự từ mạnh đến yếu dần, đó là: Thu nhận tri thức, Đào tạo nhân viên, Chiến lược và chính sách của doanh nghiệp, và VHTC; và đổi mới có tác động tích cực đến HQTC. Ngoài ra, các yếu tố khác như số năm hoạt động và quy mô của doanh nghiệp cũng có tác động đến HQTC.

So sánh với nghiên cứu trước, kết quả phân tích trên cho thấy, thu nhận tri thức có tác động mạnh nhất đến sự đổi mới. Kết quả này tương đồng với nghiên cứu của Valdez-Juárez và cộng sự (2016) cho rằng, thu nhận tri thức là một trong những thực tiễn cơ bản nhất của các doanh nghiệp trong việc quản lý đổi mới. Từ quan điểm này, các doanh nghiệp nên tập trung vào các khía cạnh của QLTT như thu nhận tri thức, phổ biến tri thức, và sử dụng tri thức để thúc đẩy sự đổi mới nhằm cải thiện HQTC. Vì vậy, các nhà quản lý, chủ sở hữu các DNVVN nên tìm cách để cải thiện tình hình thông qua việc tăng cường mạng lưới bên ngoài và đảm bảo liên lạc thường xuyên với các bên liên quan như khách hàng, nhà cung cấp, đối thủ cạnh tranh, các nhà chuyên môn, và các chuyên gia để thu nhận tri thức mới.

Về khía cạnh VHTC, kết quả này cũng tương đồng với nghiên cứu của Valdez-Juárez và cộng sự (2016) cho rằng, VHTC là yếu tố cần thiết cho việc thúc đẩy sự đổi mới trong các DNVVN. Các DNVVN cần thiết lập một nền văn hóa hữu ích và có giá trị nhằm khuyến khích chia sẻ tri thức và hợp tác trong tổ chức, đặc biệt là trong việc thiết lập một bầu không khí nội bộ để hỗ trợ cho sự đổi mới. QLTT tạo ra một nền văn hóa thúc đẩy học tập và chia sẻ tri thức, nền văn hóa như vậy khuyến khích các quy trình dựa trên tri thức, tạo ra sự chia sẻ và sử dụng tri thức, thay đổi hành vi để đổi mới và sáng tạo.

Về khía cạnh chiến lược và chính sách, kết quả này cũng tương đồng với nghiên cứu của Valdez-Juárez và cộng sự (2016) cho rằng, các DNVVN phải thành lập các chính sách và chiến lược, cả trong việc thu thập và sử dụng tri thức và triển khai VHTC, dựa trên các giá trị để tăng cường hơn nữa các hoạt động đổi mới, vì chiến lược QLTT giúp tạo ra nhiều nhân viên sáng tạo hơn, qua đó làm tăng vốn tri thức, đồng thời là những cơ chế hiệu quả để cải thiện sự đổi mới. Những thực tiễn này là một phần quan trọng trong chiến lược phân biệt, tạo ra lợi thế cạnh tranh cho các doanh nghiệp.

Riêng về khía cạnh đào tạo nhân viên, kết quả này trái ngược với nghiên cứu của ValdezJuárez và cộng sự (2016) cho rằng, không có mối quan hệ tích cực nào giữa đào tạo nhân viên và đổi mới. Sự khác biệt này được giải thích do những năm qua tại Việt Nam, Chính phủ tập trung vào các chính sách hỗ trợ nhằm thúc đẩy khu vực DNVVN phát triển, cụ thể như hỗ trợ đổi mới, sáng tạo, hiện đại hóa công nghệ, và phát triển nguồn nhân lực có đủ khả năng tham gia mạng sản xuất, chuỗi giá trị khu vực và toàn cầu; tạo nguồn vốn và đào tạo nghề cho người lao động. Thật vậy, nhiều chương trình tư vấn kiến thức về kinh doanh, công nghệ và quản lý sản xuất; tư vấn phát triển sản phẩm, phát triển thị trường, xây dựng hệ thống thông tin hỗ trợ doanh nghiệp; hợp 
tác giữa nhà trường và doanh nghiệp trong đào tạo nguồn nhân lực và đào tạo nghề cho người lao động đã được thực hiện. Về phía doanh nghiệp, nên chú trọng hơn về khía cạnh này và cần có thêm nhiều chương trình tư vấn, đào tạo chính thức và không chính thức và khuyến khích nhân việc học tập nâng cao trình độ nhằm tăng cường sự sáng tạo và chia sẻ tri thức trong nhân viên.

Xét về mối quan hệ giữa QLTT, sự đổi mới, và HQTC, kết quả của nghiên cứu này cũng tương đồng với nghiên cứu của Valdez-Juárez và cộng sự (2016) cho rằng, QLTT có ảnh hưởng đến HQTC gián tiếp thông qua sự đổi mới, các hoạt động của QLTT được thực hiện thông qua sự đổi mới trong các sản phẩm và quy trình nhằm cải thiện $\mathrm{HQTC}$ trong các DNVVN. Mặt khác, các yếu tố như loại hình doanh nghiệp và số năm hoạt động cũng có tác động đáng kể đến HQTC trong các DNVVN. Kết quả của nghiên cứu này cho thấy, nhóm doanh nghiệp vừa và siêu nhỏ có ảnh hưởng mạnh hơn các nhóm còn lại và các doanh nghiệp hoạt động từ 06 - 10 năm và từ 11 - 15 năm có ảnh hưởng nhiều nhất đến HQTC.

\section{Kết luận}

Vận tốc và tính chất năng động của thị trường đã tạo ra động lực cạnh tranh giữa nhiều doanh nghiệp để tận dụng tài sản tri thức của họ như một phương tiện tạo ra giá trị và đạt được lợi thế cạnh tranh. Trong xã hội hiện nay, tri thức trở nên quan trọng hơn, và QLTT trở thành một trong những cách tiếp cận tốt nhất để đảm bảo sự thành công của bất kỳ doanh nghiệp nào (Pham, 2017). Tập trung vào QLTT là một lĩnh vực quan trọng đối với các DNVVN. Việc quản lý tài sản tri thức có thể cung cấp cho các DNVVN các công cụ mới để tồn tại, tăng trưởng, và duy trì lợi thế cạnh tranh bền vững (Evangelista et al., 2010).

Nghiên cứu này kết luận rằng, các yếu tố thành công quan trọng của QLTT là Thu nhận tri thức, Đào tạo, Chiến lược và chính sách, và VHTC có tác động trực tiếp đến sự đổi mới và kết quả cũng cho thấy đổi mới góp phần đáng kể vào việc cải thiện HQTC của các DNVVN tại Việt Nam. Điều này có thể đạt được bằng cách xây dựng một môi trường văn hóa thúc đẩy chia sẻ và thu nhận tri thức trong nhân viên, tạo động lực và khuyến khích nhân viên nâng cao trình độ thông qua các khóa học ngắn hạn và cho phép họ tham dự các buổi hội thảo, hội nghị, và triển lãm để có được tri thức mới và tri thức mới nên được sử dụng để tăng cường các hoạt động đổi mới nhằm mang lại hiệu quả tốt hơn. Inków (2020) đã chỉ ra rằng, đổi mới và sáng tạo đã trở thành một yếu tố quan trọng quyết định sự tồn tại và phát triển của một tổ chức. Các tổ chức cần có năng lực đổi mới đủ cao để có thể phát triển và thực hiện các giải pháp mới một cách hiệu quả. Từ kết quả của nghiên cứu này có thể giúp các nhà quản lý, chủ doanh nghiệp tận dụng các nguồn tri thức sẵn có bằng cách biến chúng thành sản phẩm mới và quy trình mới để cải thiện HQTC. Mặt khác, kết quả của nghiên cứu này cũng góp phần củng cố cho các nghiên cứu trước thông qua các bằng chứng thực nghiệm. Hướng nghiên cứu tiếp theo có thể xem xét lại các biến này tại các nước đang phát triển như Việt Nam hoặc xem xét ảnh hưởng trực tiếp từ QLTT đến HQTC, hoặc tập trung vào một ngành nghề, lĩnh vực cụ thể của DNVVN.

\section{Tài liệu tham khảo}

Abdi, K., Mardani, A., Senin, A. A., Tupenaite, L., Naimaviciene, J., Kanapeckiene, L., \& Kutut, V. (2018). The effect of knowledge management, organizational culture and organizational learning on innovation in automotive industry. Journal of Business Economics and Management, 19(1), 1-19. doi:10.3846/jbem.2018.1477

Abubakar, A. M., Elrehail, H., Alatailat, M. A., \& Elçi, A. (2017). Knowledge management, decision-making style and organizational performance. Journal of Innovation \& Knowledge, 4(2), 104-114. doi:10.1016/j.jik.2017.07.003 
Al-Saifi, S. A. (2015). Positioning organisational culture in knowledge management research. Journal of Knowledge Management, 19(2), 164-189. doi:10.1108/jkm-07-2014-0287

Anderson, N., Potočnik, K., \& Zhou, J. (2014). Innovation and creativity in organizations. Journal of Management, 40(5), 1297-1333. doi:10.1177/0149206314527128

Asgarian, N. (2012). Knowledge management capacity and innovation performance. Management Science Letters, 2(8), 2739-2746. doi: 10.5267/j.msl.2012.10.015

Augier, M., \& Teece, D. J. (2009). Dynamic capabilities and the role of managers in business strategy and economic performance. Organization Science, 20(2), 410-421. doi:10.1287/orsc. 1090.0424

Bozbura, F. T. (2007). Knowledge management practices in Turkish SMEs. Journal of Enterprise Information Management, 20(2), 209-221. doi:10.1108/17410390710725788

Byukusenge, E., \& Munene, J. C. (2017). Knowledge management and business performance: Does innovation matter? Cogent Business \& Management, 4(1), 1-18. doi:10.1080/23311975.2017.1368434

Byukusenge, E., Munene, J., \& Orobia, L. (2016). Knowledge management and business performance: Mediating effect of innovation. Journal of Business and Management Sciences, 4(4), 82-92. doi:10.12691/jbms-4-4-2

Cantú, L. Z., Criado, J. R., \& Criado, A. R. (2009). Generation and transfer of knowledge in IT-related SMEs. Journal of Knowledge Management, 13(5), 243-256. doi:10.1108/13673270910988088

Chawla, D., \& Joshi, H. (2010). Knowledge management practices in Indian industries: A comparative study. Journal of Knowledge Management, 14(5), 708-725. doi:10.1108/13673271011074854

Chen, Y. S., Lin, M. J. J., \& Chang, C. H. (2009). The positive effects of relationship learning and absorptive capacity on innovation performance and competitive advantage in industrial markets. Industrial Marketing Management, 38, 152-158. doi:10.1016/j.indmarman.2008.12.003

Chen, Y. Y., \& Huang, H.-L. (2012). Knowledge management fit and its implications for business performance: A profile deviation analysis. Knowledge-based Systems, 27, 262270. doi:10.1016/j.knosys.2011.11.012

Cohen, W. M., \& Levinthal, D. A. (2000). Absorptive capacity: A new perspective on learning and innovation. Strategic Learning in a Knowledge Economy, 39-67. doi:10.1016/b978-07506-7223-8.50005-8

Darroch, J. (2003). Developing a measure of knowledge management behaviours and practices. Journal of Knowledge Management, 7(5), 41-54. doi:10.1108/13673270310505377

Durst, S., \& Edvardsson, I. R. (2012). Knowledge management in SMEs: A literature review. Journal of Knowledge Management, 16, 879-903. doi:10.1108/13673271211276173

Evangelista, P., Esposito, E., Lauro, V., \& Raffa, M. (2010). The adoption of knowledge management systems in small firms. Electronic Journal of Knowledge Management, 8(1), 33-42.

Hair, J., Black, W. C., Babin, B. J., \& Anderson, R. E. (2010). Multivariate data analysis (7th ed.). Upper saddle River, NJ: Pearson Education.

Halila, F., \& Rundquist, J. (2011). The development and market success of eco-innovations. European Journal of Innovation Management, 14(3), 278-302. doi:10.1108/14601061111148807

Hoang, T., \& Chu, N. N. M. (2008). Phân tích dĩ liệu NC với SPSS [Analyze NC data with SPSS]. Ho Chi Minh, Vietnam: Nhà xuất bản Thống Kê. 
Inków, M. (2020). Organizational innovation capability as a result of knowledge management processes - A literature review. Management (Sciendo), 24(1), 143-156. doi:10.2478/manment-2019-0040

Kalay, F., \& Lynn, G. S. (2015). The impact of strategic innovation management practices on firm innovation performance. Research Journal of Business and Management - (RJBM), 2(3), 412-429. doi:10.17261/Pressacademia.2015312989

Kim, D.-Y., Kumar, V., \& Kumar, U. (2012). Relationship between quality management and innovation. Journal of Operations Management, 30(4), 295-315. doi:10.1016/j.jom.2012.02.003

Lee, H., \& Choi, B. (2003). Knowledge management enablers, processes, and organizational performance: An integrative view and empirical examination. Journal of Management Information System, 20(1), 179-228. doi:10.1080/07421222.2003.11045756

Mardani, A., Nikoosokhan, S., Moradi, M., \& Doustar, M. (2018). The relationship between knowledge management and innovation performance. The Journal of High Technology Management Research, 29(1), 12-26. doi:10.1016/j.hitech.2018.04.002

Nikabadi, M. S., Bagheri, S., \& Mohammadi-Hoseini, S. A. (2016). Effects of knowledge management strategy and organizational learning capability on innovation-driven performance in an oil company. Knowledge Management \& E-Learning, 8(2), 334-355. doi:10.34105/j.kmel.2016.08.022

Nonaka, I., \& Takeuchi, H. (1995). The knowledge creating company: How Japanese companies create the dynamics of innovation. New York, NY: Oxford University Press.

Nunnally, J. (1978). Psychometric theory. New York, NY: McGraw Hill.

Paez-Logreira, H., Zamora-Musa, R., \& Velez-Zapata, J. (2016). Relation analysis of knowledge management, research, and innovation in university research groups. Journal of Technology Management \& Innovation, 11(4), 5-11. doi:10.4067/s0718-27242016000400002

Pannu, H. (2017). The impact of knowledge management infrastructure on organizational performance in SMEs. International Journal of Human Resource \& Industrial Research, 4(2), 26-31.

Park, Y., Shin, J., \& Kim, T. (2010). Firm size, age, industrial networking, and growth: A case of the Korean manufacturing industry. Small Business Economics, 35(2), 153-168. doi:10.1007/s11187-009-9177-7

Pelser, T. (2014). The affect of innovation strategies and their connect to company performance. Mediterranean Journal of Social Sciences, 5(9), 60-68. doi:10.5901/mjss.2014.v5n9p60

Pham, Q. T. (2016). Giáo trình Quản lý Tri thúc [Knowledge management curriculum]. Hanoi, Vietnam: NXB Xây Dựng.

Pham, Q. T. (2017). A knowledge management approach for ensuring the success of IT industries in Vietnam. Hauppauge, NY: Nova Science Publishers, Inc.

Pham, Q. T., \& Le, M. H. (2018). The impact of knowledge management on innovation performance of small and medium enterprises - An empirical study in Lam Dong province. Journal of Science Ho Chi Minh City Open University, 8(3), 117-132. doi:10.46223/HCMCOUJS.econ.en.8.2.168.2018

Pham, Q. T., \& Nguyen, D. T. (2017). An empirical investigation of knowledge management in Vietnamese SMEs. 17th International Conference on Computational Science and Its Applications (ICCSA), IEEE, 1-6. doi:10.1109/ICCSA.2017.8000016 
Plessis, M. (2007). The role of knowledge management in innovation. Journal of Knowledge Management, 11(4), 20-29. doi:10.1108/13673270710762684

Polanyi, M. (1966). The tacit dimension. London, UK: Routledge \& Kegan Paul.

Rashdi, M. A., Akmal, S. B., \& Al-shami, S. A. (2019). Knowledge management and organizational performance: A research on systematic literature. International Journal of Innovative Technology and Exploring Engineering (IJITEE), 8(6S4), 757-762. doi:10.35940/ijitee.F1153.0486S419

Rosenbusch, N., Brinckmann, J., \& Bausch, A. (2011). Is innovation always beneficial? A metaanalysis of the relationship between innovation and performance in SMEs. Journal of Business Venturing, 26(4), 441-457. doi:10.1016/j.jbusvent.2009.12.002

Roxas, B., Battisti, M., \& Deakins, D. (2014). Learning, innovation and firm performance: Knowledge management in small firms. Knowledge Management Research \& Practice, 12, 443-453. doi:10.1057/kmrp.2012.66

Sáenz, J., Aramburu, N., \& Rivera, O. (2009). Knowledge sharing and innovation performance: A comparison between high- tech and low- tech companies. Journal of Intellectual Capital, 10, 22-36. doi:10.1108/14691930910922879

Serban, A. M., \& Luan, J. (2002). Overview of knowledge management. New Direction for Institutional Research, 113, 5-16.

Serenko, A. (2013). Meta-analysis of scientometric research of knowledge management: Discovering the identity of the discipline. Journal of Knowledge Management, 17(5), 773812. doi:10.1108/jkm-05-2013-0166

Sethibe, T., \& Steyn, R. (2016). Innovation and organisational performance: A critical review of the instruments used to measure organisational performance. The Southern African Journal of Entrepreneurship and Small Business Management, 8(1), 12. doi:10.4102/sajesbm.v8i1.50

Sørensen, J. B., \& Stuart, T. E. (2000). Aging, obsolescence, and organizational innovation. Administrative Science Quarterly, 45(1), 81-112. doi:10.2307/2666980

Suhag, A. K., Solangi, S. R., Larik, R. S. A., Lakho, M. K., \& Tagar, A. H. (2017). The relationship of innovation with organizational performance. International Journal of Research - Granthaalayah, 5(2), 292-306. doi:10.5281/zenodo.345736

Torabi, F., \& El-Den, J. (2017). The impact of knowledge management on organizational productivity: A case study on koosar bank of Iran. Procedia Computer Science, 124, 300310. doi:10.1016/j.procs.2017.12.159

Valdez-Juárez, L. E., García-Pérez de Lema, D., \& Maldonado-Guzmán, G. (2016). Management of knowledge, innovation and performance in SMEs. Interdisciplinary Journal of Information, Knowledge, and Management, 11, 141-176. doi:10.28945/3455

Velu, C. (2015). Knowledge management capabilities of lead firms in innovation ecosystems. AMS Review, 5(3/4), 123-141. doi:10.1007/s13162-015-0068-6

Wong, K. Y., \& Aspinwall, E. (2005). An empirical study of the important factors for knowledge-management adoption in the SME Sector. Journal of Knowledge Management, 9(3), 64-82. doi:10.1108/13673270510602773

Zheng, W., Yang, B., \& McLean, G. N. (2010). Linking organizational culture, structure, strategy, and organizational effectiveness: Mediating role of knowledge management. Journal of Business Research, 63(7), 763-771. doi:10.1016/j.jbusres.2009.06.005 